\title{
A novel method of imaging pancreatic cancer cells and precursors in mice could lead to early diagnosis
}

Pancreatic cancer cells and precursor lesions in mice can be visualized with a novel imaging method that uses a labelled toxin fragment from Clostridium perfringens, according to new research. "Given the appalling prognosis of pancreatic cancer, partly due to late diagnosis, there is an urgent need for tools to aid early detection," says Thomas Gress, corresponding author.

Claudin-4 is a tight-junction protein on epithelial cells, originally described as a receptor for $C$. perfringens enterotoxin (CPE). Pancreatic cancer cells upregulate expression of claudin-4 and full-length CPE has an acute cytotoxic effect, which could potentially be used therapeutically. Toxicity is mediated by the $\mathrm{N}$-terminal half of CPE and the $C$-terminal end binds to claudin-4. The researchers conjugated a fluorochrome tracer to a nontoxic $C$-terminal fragment (C-CPE) to create a marker with high-affinity for claudin-4.
Conjugated C-CPE bound to claudin- $4^{+}$ pancreatic cancer cells with high affinity, but there was little binding to

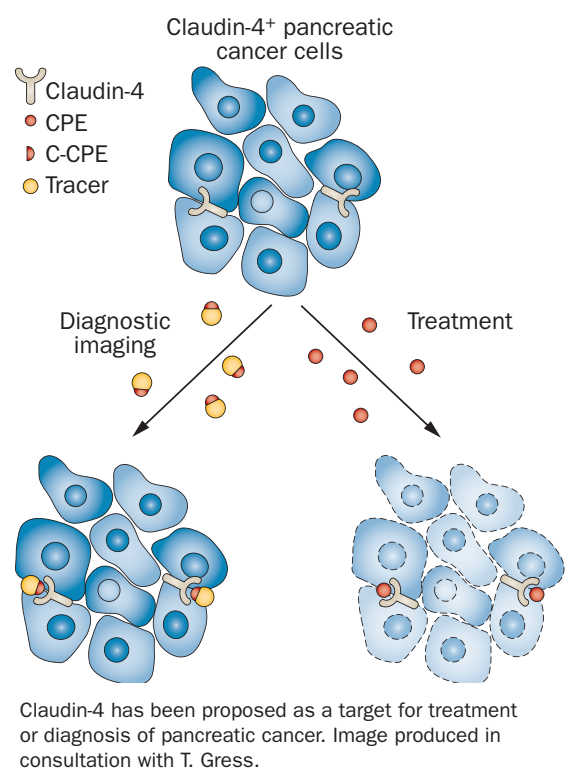

claudin- $4^{-}$cells in vitro. Pancreatic tumour cells, precursor lesions and hepatic metastases were visualized by $2 \mathrm{D}$ planar fluorescence reflectance imaging and 3D fluorescence-mediated tomography after conjugated C-CPE was injected into mice. Tumours had markedly higher accumulation of fluorochome than normal tissue.

"Tracer-conjugated C-CPE and novel imaging methods enable noninvasive visualization of claudin $-4^{+}$mouse pancreatic tumours and precursor lesions that could be a promising modality for early diagnosis," explains Gress. The researchers are trying to link $\mathrm{C}$-CPE to tracers such as ${ }^{18} \mathrm{~F}$ that can be detected by scanners used in routine clinical practice.

Andy McLarnon

Original article Neesse, A. et al. Claudin-4-targeted optical imaging detects pancreatic cancer and its precursor lesions. Gut doi:10.1136/gutjnl-2012-302577 